\title{
Laparoscopic Repair of Diastasis Recti: A Case Report and Literature Review
}

\author{
Woohyung Lee, M.D. ${ }^{1}{ }^{2}$, Jin-Kyu Cho, M.D. ${ }^{1}$, Jae Yool Jang, M.D. ${ }^{1}$, Soon-Chan Hong, M.D., Ph.D. ', Chi-Young Jeong, M.D., Ph.D. ${ }^{1}$ \\ 'Department of Surgery, Gyeongsang National University Hospital, Gyeongsang National University Postgraduate School of Medicine, Jinju, ${ }^{2}$ Division of \\ Hepatobiliary and Pancreatic Surgery, Department of Surgery, Asan Medical Center, University of Ulsan College of Medicine, Seoul, Korea
}

\begin{abstract}
Diastasis recti is a state with separated aponeurosis between two recti caused by weakening of the intercrossing fibers in the linea alba and it causes abdominal protrusion. Common causes comprised of increased intraabdominal pressure, or congenital weakening of myoaponeurotic layer. We describe a patient who underwent laparoscopic repair of diastasis recti. A 30-year-old woman was referred to our outpatient department for an abdominal mass that had appeared 1 year earlier. Physical examination revealed an abdominal wall defect along the midline and computed tomography showed thinning and stretching of the linea alba. The patient underwent laparoscopic repair for diastasis recti. The stretched linea alba was approximated using interrupted sutures from the epigastrium to the suprapubic area. A dual mesh was applied below the peritoneum to prevent recurrence. The patient was discharged without complications, and was followed up for 1 year without recurrence. Laparoscopic repair could be a considerable is a treatment modality for diastasis recti.
\end{abstract}

Keywords: Laparoscopy, Diastasis recti, Linea alba, Repair

This is an Open Access article distributed under the terms of the Creative Commons Attribution Non-Commercial License (http:// creativecommons.org/licenses/by-nc/4.0/) which permits unrestricted non-commercial use, distribution, and reproduction in any medium, provided the original work is properly cited.
Received September 28, 2018

Revised December 23, 2018

Accepted January 7, 2019

Corresponding author

Chi-Young Jeong

Department of Surgery, Gyeongsang

National University Hospital,

Gyeongsang National University

Postgraduate School of Medicine, 79

Gangnam-ro, Jinju 52723, Korea

Tel: $+82-55-750-8096$

Fax: +82-55-750-9244

E-mail: drjcy@hanmail.net

ORCID:

https://orcid.org/0000-0003-4121-6695

Copyright $\odot 2019$ The Journal of Minimally Invasive Surgery. All rights reserved.

\section{INTRODUCTION}

Diastasis recti is a state in which the rectus abdominis has separated, and is related to thinning and stretching of the linea alba. ${ }^{1}$ Affected patients usually display an abdominal protrusion. The most common acquired causes are pregnancy or obesity. Recently, some surgeons have reported cosmetic advantages of minimally invasive treatment compared with the conventional approach for treating diastasis recti. ${ }^{2}$ In this case report and video, we describe laparoscopic repair of diastasis recti.

\section{CASE REPORT}

A 30-year-old woman was referred to our outpatient department because of an abdominal protrusion that had persisted for 1 year. The patient had iron deficiency anemia, which was related to menorrhagia. She underwent laparoscopic cystectomy for a ruptured right ovarian cyst a month ago and delivered a child 1 year before referral. Physical examination revealed an abdominal wall defect along the midline. Abdominal computed tomography (CT) revealed thinning and stretching of the linea alba. The inter-recti distance was $6 \mathrm{~cm}$ (Fig. 1).

Laparoscopic repair was performed on 15 April 2016. Under general anesthesia, the patient was placed in the Trendelen- 

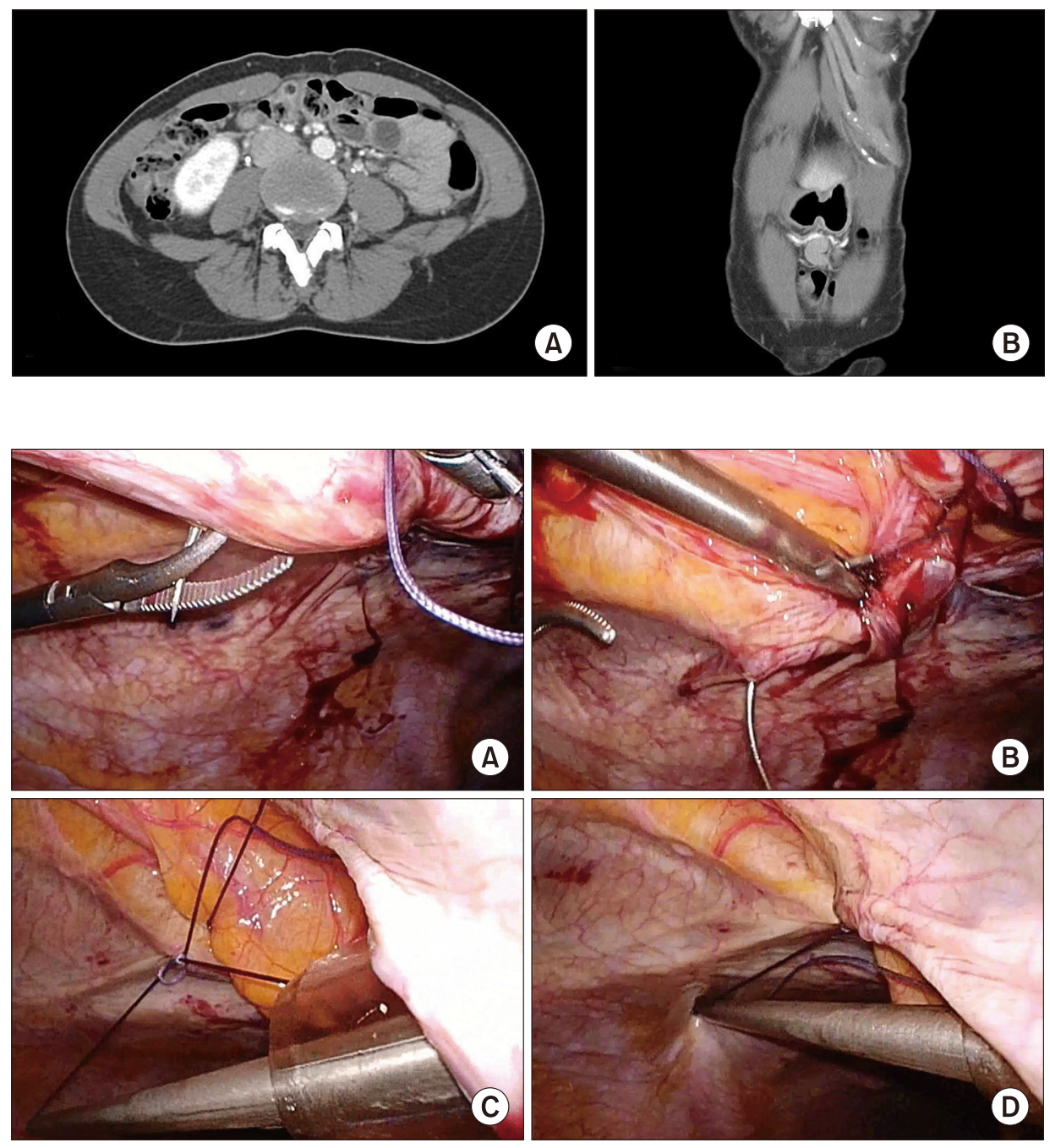

Fig. 1. Abdominal computed tomography revealed thinning and stretching of the linea alba. The inter-recti distance was 6 $\mathrm{cm}$. (A) Axial view, (B) coronal view.
Fig. 2. The operator was stood at the left side of the patients. The separated rectus muscle and linea alba were sutured in each side using 1-0 absorbable thread $(A, B)$ and approximated from the epigastrium to the suprapubic area (C, D). burg position with the surgeon and scopist standing on the patient's left side. One 5-mm trocar and two $12-\mathrm{mm}$ trocars were placed into the left abdomen for surgical devices and the camera. The intraabdominal pressure was maintained at $\sim 10 \mathrm{mmHg}$. The separated fascia with rectus abdominis were approximated using interrupted absorbable multifilament sutures placed from the epigastrium to the suprapubic area (Fig. 2). After approximation, a dual mesh was applied to prevent recurrence of the hernia. The dual mesh comprised of polypropylene on one side and expanded polytetrafluoroethylene on the other side. Before inserting the dual mesh, we applied non-absorbable monofilament sutures along each edge to attach it to the peritoneum and the sutured mesh was placed below the midline. Each thread was retrieved through different holes using a Carter-Thomason needle and was tied below the skin. A laparoscopic tacker was applied among the suture line (Fig. 3). The patient was discharged on postoperative day 4 without postoperative complications. She has not exhibited any signs of recurrence at her follow-up visits for 1 year after surgery.

\section{DISCUSSION}

Diastasis recti is a condition with separated aponeurosis between two recti caused by weakening of the intercrossing fibers in the linea alba. ${ }^{1}$ Common causes include pregnancy, obesity, excessive exercise, or congenital disease, which increase the intraabdominal pressure, or congenital weakening of the myoaponeurotic layer. ${ }^{3}$ The inter-recti distance is the main criterion for diastasis recti and normal width or the linea was $22 \mathrm{~mm}$ around umbilicus in nulliparous women. ${ }^{4}$

Although patients are usually referred for treatment because of abdominal protrusion, the risk of herniation or strangulation of the intestine is low and surgical treatment is usually performed for cosmetic purposes. ${ }^{3}$ Surgical treatment may involve open or laparoscopic procedures. Open procedures in- 

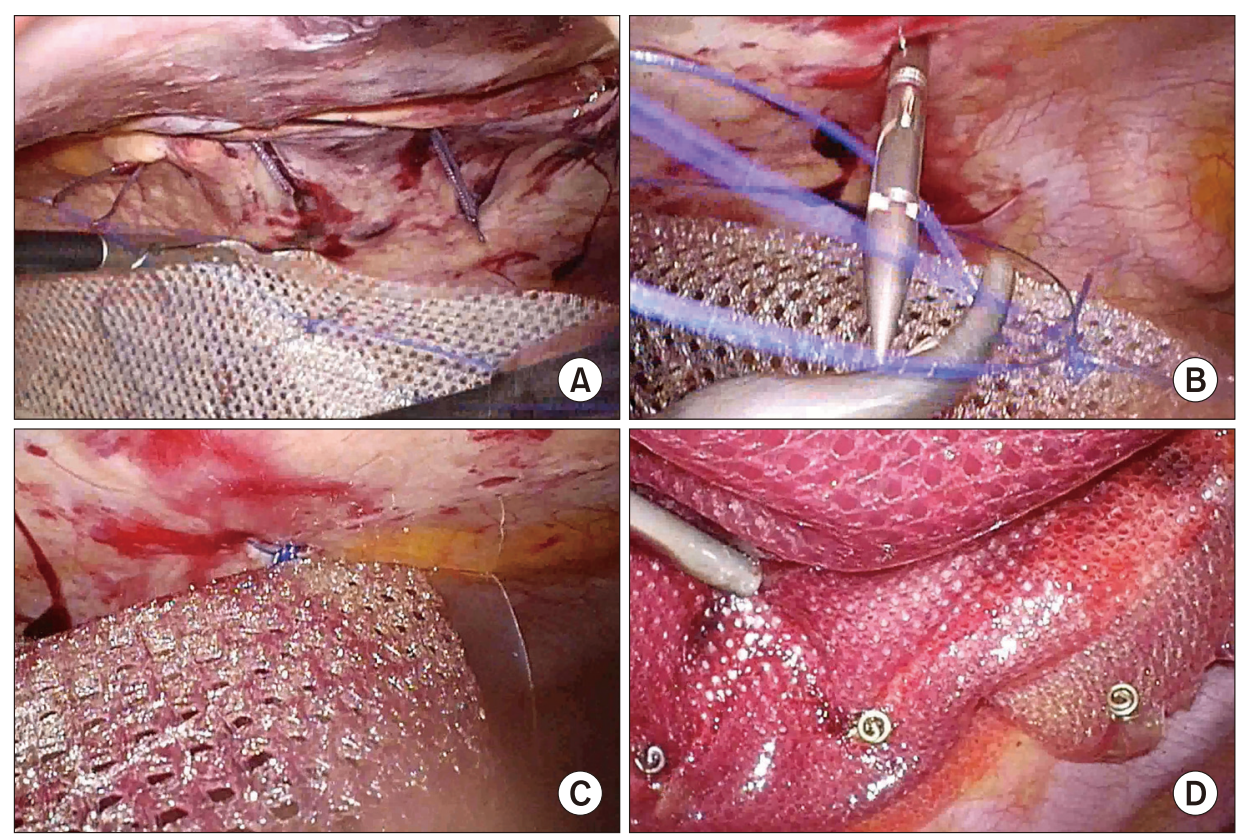

Fig. 3. A dual mesh was attached to the peritoneum to prevent recurrence. Before inserting the dual mesh, nonabsorbable monofilament sutures were applied along each edge of the mesh (A). Each thread was retrieved through different holes using a Carter-Thomason needle and was tied below the skin (B, C). A laparoscopic tacker was applied among the suture line (D).

clude long incision, liposuction, plication of both aponeuroses, and application of a mesh. ${ }^{5,6}$ Common postoperative complications of these open procedures include seroma, hematoma, minor skin necrosis, wound infection, and postoperative pain. Several surgeons have reported that the supra-aponeurotic endoscopic approach provided a good cosmetic appearance and had a low rate of recurrence. ${ }^{7-9}$

Laparoscopic procedures were introduced in recent years, and several surgeons have compared them with open procedures. Laparoscopic repair generally involves one layer of interrupted sutures and application of a composite mesh., ${ }^{2,10}$ Seroma and postoperative pain are the most common postoperative complications of laparoscopic repair. To reduce postoperative seroma, Palanivelu et al. ${ }^{2}$ proposed the Venetian blinds technique, which involves multiple sutures placed along the stretched linea alba. One study reported a lower rate of complications with laparoscopic procedures than with open procedures. ${ }^{10}$ However, because of the rarity of diastasis recti, there is no definite conclusion on which procedure offers better outcomes. Furthermore, recent studies showed long term complication related with mesh or tacker after surgery for incisional hernia and these results affect operative strategy. The operative results included intracorporeal sutures with mesh are needed to reported.

In conclusion, minimally invasive procedures may be a considerable treatment modality for diastasis recti. However, further analysis using large data will confirm the feasibility of laparoscopic repair for diastasis recti.

\section{AUTHORS' CONTRIBUTIONS}

Conceptualization: WHL, CYJ. Formal analysis: JKC. Methodology: WHL, JYJ. Writing-original draft: WHL, SCH. Writing-review and editing: CYJ, SCH.

\section{ORCID}

Woohyung Lee, https://orcid.org/0000-0002-8119-6943

Jin-Kyu Cho, https://orcid.org/0000-0002-6877-7620

Jae Yool Jang, https://orcid.org/0000-0002-6070-4398

Soon-Chan Hong, https://orcid.org/0000-0003-4499-8741

Chi-Young Jeong, https://orcid.org/0000-0003-4121-6695

\section{CONFLICT OF INTEREST}

None.

\section{FUNDING}

None.

\section{ACKNOWLEDGMENTS}

None.

\section{REFERENCES}

1) Akram J, Matzen SH. Rectus abdominis diastasis. J Plast Surg Hand Surg 2014;48:163-169. 
2) Palanivelu C, Rangarajan M, Jategaonkar PA, Amar V, Gokul KS, Srikanth B. Laparoscopic repair of diastasis recti using the "Venetian blinds' technique of plication with prosthetic reinforcement: a retrospective study. Hernia 2009;13:287-292.

3) Hickey F, Finch JG, Khanna A. A systematic review on the outcomes of correction of diastasis of the recti. Hernia 2011;15:607614.

4) Beer GM, Schuster A, Seifert B, Manestar M, Mihic-Probst D, Weber SA. The normal width of the linea alba in nulliparous women. Clin Anat 2009;22:706-711.

5) Gama LJM, Barbosa MVJ, Czapkowski A, Ajzen S, Ferreira LM, Nahas FX. Single-Layer Plication for Repair of Diastasis Recti: The Most Rapid and Efficient Technique. Aesthet Surg J 2017;37:698-705.

6) Brauman D, Capocci J. Liposuction abdominoplasty: an advanced body contouring technique. Plast Reconstr Surg 2009;124:16851695.

7) Bellido Luque J, Bellido Luque A, Valdivia J, et al. Totally endoscopic surgery on diastasis recti associated with midline hernias. The advantages of a minimally invasive approach. Prospective cohort study. Hernia 2015;19:493-501.

8) Chang CJ. Assessment of videoendoscopy-assisted abdominoplasty for diastasis recti patients. Biomed J 2013;36:252-256.

9) Lockwood T. Rectus muscle diastasis in males: primary indication for endoscopically assisted abdominoplasty. Plast Reconstr Surg 1998;101:1685-1691; discussion 1692-1684.

10) Zukowski ML, Ash K, Spencer D, Malanoski M, Moore G. Endoscopic intracorporal abdominoplasty: a review of 85 cases. Plast Reconstr Surg 1998;102:516-527. 